\title{
Apexification of Maxillary Incisor in Adult Patient With Cleft Lip and Palate
}

\author{
Sarah Eveline Desita Nainggolan ${ }^{1, *}$, Tunjung Nugraheni ${ }^{2}$, Margareta Rinastiti ${ }^{2}$ \\ ${ }^{1}$ Post-graduate Student, Department of Conservative Dentistry, Faculty of Dentistry Universitas Gadjah \\ Mada, Yogyakarta, Indonesia \\ ${ }^{2}$ Department of Conservative Dentistry, Faculty of Dentistry Universitas Gadjah Mada, Yogyakarta, Indonesia \\ *Corresponding author.Email :drg.saraheveline@gmail.com,drgtunjungnugraheni@ugm.ac.id,rinastiti@ugm.ac.id
}

\begin{abstract}
Introduction. Cleft lip and palate are congenital abnormalities that caused by many factors. Dental abnormalities in patients with cleft lip and palate were begun during eruption phase such as caries, crown and root deformity, abnormalities number of teeth and the relationship between maxilla and mandibula. Interdisciplinary treatment are required to gain optimum results for patients with clefts. Apexification followed by proper restoration are needed to correct a necrotic tooth with an open apex in cleft lip and palate patients. The aim of this paper is to report treatment of necrotic tooth with open apex in a cleft lip and palate patient. Case report. A 23-year-old female patient was referred to Department of Conservative Dentistry by orthodontist to treat her central incisor maxilla. The patient had a history of cleft lip and palate and had been wearing orthodontic braces for 3 years. Tooth 11 was asymptomatic, periapical radiograph showed radiolucent area on the apical region with open apex. The diagnosis was necrotic pulp with open apex. Apexification treatment using Mineral Trioxide Aggregate followed by fiber post and indirect composite crown were carried out. Indirect composite crown were made on tooth 21 for an aesthetic purpose. The patient returned for follow-up after 6 months with no complaints. Both clinical finding and radiograph show normal tissues and the tooth function normally. Conclusion. The apexification followed by indirect composite crown are the right treatment for treating maxillary central necrotic incisor with open apex in the cleft lip and palate patient.
\end{abstract}

Keywords: Open apex, Dental anomalies, Labiopalatoschisis

\section{INTRODUCTION}

Cleft lip and palate is the most common craniofacial congenital abnormalities and its etiology caused by many factor. It is known that both genetics and environment have role in the condition. The most considered environment factors to the risk of cleft lip and palate are maternal cigarette smoking, alcohol consumption and multivitamin used [1]. Patients with cleft lip and palate suffer a multitude of problems related to function and aesthetics. Cleft lip and palate is accompanied by a wide variety of dental anomalies and in the long term will impact on facial anatomy and selfesteem [2].

Studies have shown that cleft lip and palate may affect both permanent and deciduous teeth and more frequently on the cleft side. The common dental anomalies that found in cleft lip and palate patients are: agenese/multiple missing teeth/hypodontia (the maxillary lateral incisors are the most susceptible); microdontia; ectopic teeth; supernumerary teeth; impaction; maxillary canines and premolars transposition; crown and root malformation; multiple decayed teeth and delayed development [3]. The management of cleft lip and palate patient requires prolonged orthodontic treatment and an interdisciplinary approach to providing optimal function, esthetics, and stability [4].

Root malformation such as an open apical root is one of the most common dental anomalies in cleft lip and palate patients. The obturation and good apical seal are difficult to achieve because of the lack of an apical stop. Apexification has been defined as the nonsurgical condensation of a biocompatible material into the apical end of root canal. Mineral trioxide aggregate provides an excellent biological seal and good compressive strength. Mineral trioxide aggregate is a hydrophilic material that has a 3-hour setting time in the presence of moisture [5]. Apexification followed by proper restoration are needed to treat a necrotic tooth with an open apex in cleft lip and palate patients and reach aesthetical and functional rehabilitation [6]. The 
aim of this paper is to report the treatment of necrotic tooth with open apex in a cleft lip and palate patient and follow-up results consecutively treated teeth which were managed with an MTA.

\section{CASE REPORT}

\subsection{Case Presentation}

A 23-year-old female patient was referred to Department of Conservative Dentistry Universitas Gadjah Mada Dental Hospital by orthodontist to treat her central incisor maxilla. The patient had a history of cleft lip and palate and had been wearing orthodontic braces for 3 years. Patient did not have any systemic disease. No significant family history was revealed. Extraoral findings were unremarkable. Dental history of patient revealed caries occurred in right central incisor maxilla. She did not feel any pain neither unpleasant feeling on the affected tooth. Clinical examination was cavity on palatal area (Figure 1) and asymetrical height of margin gingiva (Figure 2). Tooth \#11 was asymptomatic, vitality test showed negative result with no tenderness on percussion, and periapical radiograph showed radiolucent area on the apical region with open apex (Figure 3). Based on examination tooth \#11 was diagnosed necrotic pulp with open apex.

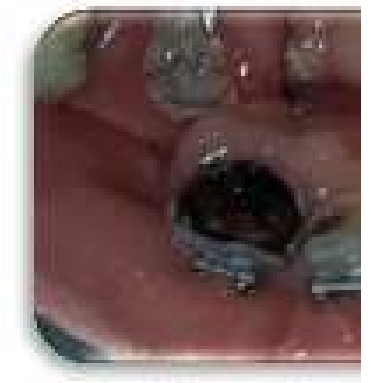

Figure 1. Cavity on the palatal side of \#11
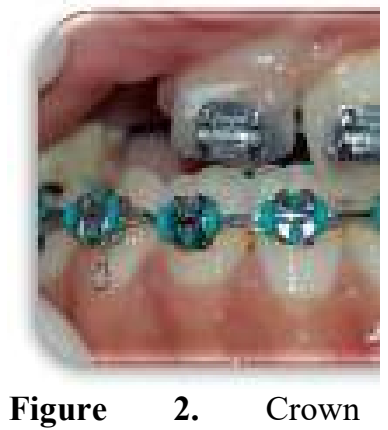
malformation of tooth $\# 11$ and \#21 from labial side

\subsection{Investigations}

An intraoral periapical radiograph had showed incomplete root formation with wide open apices in right upper central incisors. There was radiolucent area under apical root which showed no alveolar bone on apical area (Figure 3). Electric pulp test was carried out which showed that the upper right central incisor \#11 was non-vital.

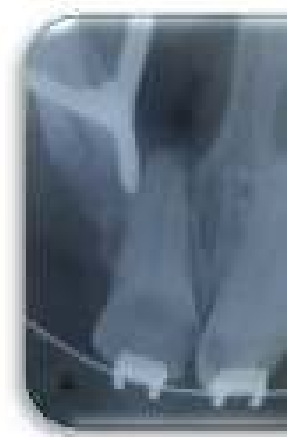

Figure 3. Preoperative diagnostic radiograph

\subsection{Treatment}

Based on clinical examination, there was an asymetrical height of margin gingiva on labial area. Crown lengthening was done before the root canal preparation. Crown lengthening was done on labial area of margin gingiva \#11 due to aesthetic reason and functional reason on the palatal area to provide the ferrule effect for the post fiber (Figure 4). One weeks later, the crown lengthening's wound healed. The tooth was isolated by a rubber dam, access was made. Working length was determined by apex locator and was established radiographically (Figure 5). Cleaning and shaping was done with circumferential filing using the International Organization for Standardization (ISO) 80 K-file during the same visit. Copious irrigation with saline and $2.5 \%$ sodium hypochlorite was used after each instrument. A volume of $17 \%$ ethylenediaminetetraacetic acid (EDTA) solution was used for smear layer removal. After drying the canals with paper points, calcium hydroxide was applied as intracanal medicament and the cavity was sealed with temporary restoration.

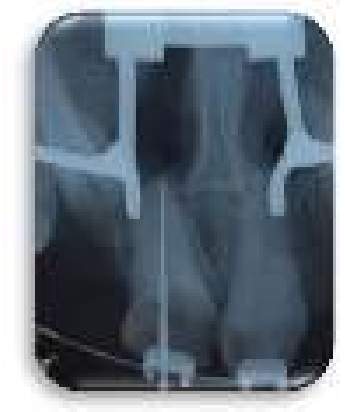

Figure 5. Working length determination radiograph

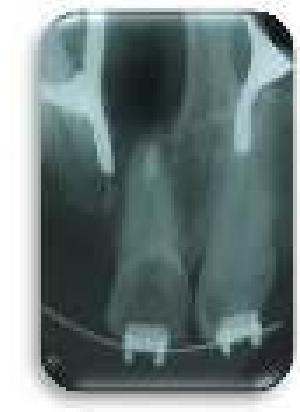

Figure 6. placement

One weeks later, tooth was again accessed under rubber dam isolation, and copious amount of normal saline was used to remove any remnants of the calcium hydroxide medicament. Canal was thoroughly dried with absorbent paper points. Apical plug of about $4 \mathrm{~mm}$ 
of MTA was placed and gently condensed using hand plugger and confirmed radiographically (Figure 6). A sterile cotton pellet moistened with sterile water was placed over the canal orifice and the access cavity was sealed with temporary restoration.

Next day, the patient was recalled for her aesthetic rehabilitation. There was minimum crown structure left, a fibre post and core was placed in the tooth \#11. Crown preparations were done not only on the tooth \#11 but also \#21 for an aesthetic purpose (Figure 7). After determine the tooth color A2, the gingival retraction cord was placed inside the gingival sulcus and impression was taken. Composite crowns were cemented on the fifth visit, 3 days after the impression was taken (Figure 8).

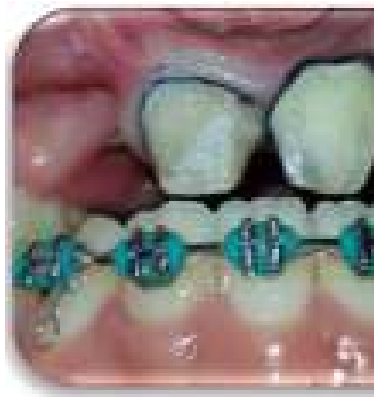

Figure 7. Crown preparation of central upper incisor after fiber post placement of tooth \#11

A month later the patient came for follow-up and no complaints were noted. Patient was recalled again after 6 months for check up, patient finished her orthodontic treatment and continued denture to replace the missing teeth (Figure 9). There was still no complaints on the tooth \#11. Periapical radiograph showed good adaptation of the restoration and no sign of apical lesion (Figure 10).
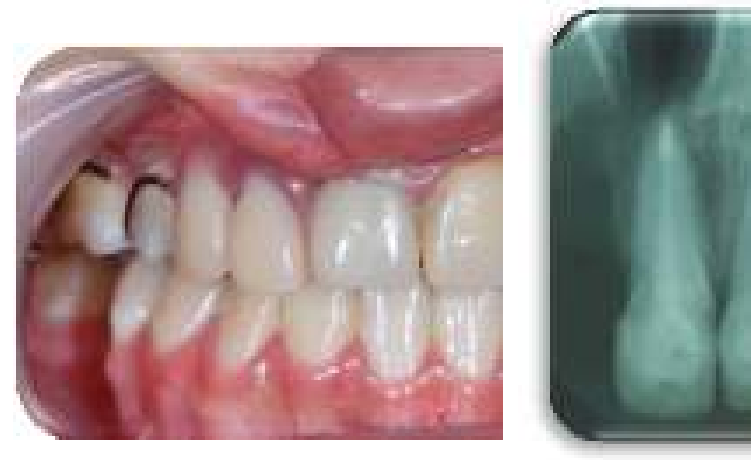

Figure 9. Tooth \#11 6 months after apexification (clinical view)
Figure 10.

Periapical radiograph 6 months after apexification

\section{DISCUSSION}

Cleft lip and palate is a congenital disorder that occurs in the mouth and lips are. The etiology of cleft lip and palate caused by many factor including genetics and environment [1]. Cleft lip and palate cause a variety of problems related to the oral cavity, talking, hearing and may also affect the number, size, shape and position of the primary teeth and permanent teeth [7]. Cleft lip and palate has a quite complex problem and requires a multi interdisciplinary studies [8]. Dental anomalies that can be found in cleft lip and palate patients are crown and root malformation, multiple decayed teeth, and also delayed development [3]

Cleft lip and palate in this case repot had been wearing orthodontic braces for 3 years. Based on her dental history and radiograph, the cavity on her tooth and the crown and root malformation on tooth \#11 occured before before her orthodontic treatment. Patients with cleft lip and palate generally have poorer oral hygiene and higher susceptibility to caries because of the difficulty in achieving adequate plaque control associated with dental anomalies and defects from lips or palate. The risk of caries increases when fixed appliance orthodontic treatment applied because of the increased accumulation of plaque around the attachments and difficulty in cleaning [9]

Multiple decay teeth and delayed development of tooth are common dental anomalies that can be found in cleft lip and palate patients [3]. Necrose pulp during root formation can cause failure root formation. The root canal become wide and open apical root so that it will be very vulnerable to fracture root or crown. Among the possible treatments to perform in a permanent tooth with open apical root that need endodontic therapy, apexification is a procedure of induction of a calcified apical barrier in the apical zone of an incompletely formed root, in which the pulp is diagnosed as necrotic [10]. Apexification is an initial treatment that must be done with the aim of getting apical calcific barrier before restoration.

Conventional using calcium hydroxide has many limitation such as variable treatment time ranging from 5 months to 20 months, poor patient compliance with follow-up due to the extended treatment time, apical closure in relationship to treatment time is unpredictable, and an increased risk of tooth fracture [11]. Mineral trioxide aggregate (MTA) is used as an apical barrier for teeth with immature apices, It also has a shorter treatment time compared with calcium hydroxide, and a more predictable time to apical closure. However, MTA has some limitations such as nonreinforcement of root canal dentin and a higher cost than calcium hydroxide [12]. Apexification using MTA can be done in two visits until the final restoration is made so that it prevents fractures and can provide advantages in reducing the amount of exposure radigraphic and cooperative enhancement of patients. 
The apexification using MTA procedure begins with debridement and intracanal calcium hydroxide medicament for 7 days. MTA material can stimulates calcific barriers formation and apical healing so that it forms apical density and restoration can be done immediately to prevent fracture in immature teeth. ${ }^{12}$ In this case fiber post was placed into root canal to strengthen root canal wall without reduce the thicknesses of canal wall. Composite crown restoration was placed not only on the tooth \#11 but also \#21 to correct the aestethic of central upper incisor. Cleft lip and palate need a multi interdisciplinary studies to improve the result. After finish endodontic and orthodontic treatment, prosthodontic treatment was done to replace the missing tooth on the cleft side and improve the patient self esteem.

\section{CONCLUSION}

The management of cleft lip and palate patient requires a multiple treatment to providing optimal function and esthetics. There are common dental anomalies that found in cleft lip and palate patients such as crown and root malformation. Root development of the central incisor was more influenced by the cleft side. MTA apical plug method is an effective and efficient method because of the less requirement of treatment time. MTA can shorten the time of visit with formation apical barrier which stimulates healing and can be continued immediately with the final restoration.

\section{ACKNOWLEDGMENTS}

We are grateful to orthodontist Retno Iswati, Department of Orthodontics, , Faculty of Dentistry Universitas Gadjah Mada for kindly cooperation.

\section{REFERENCES}

[1] Alexandre R. Vieira. Genetics and Environmental Factors in Human Cleft Lip and Palate. Cobourne MT (ed): Cleft Lip and Palate. Epidemiology, Aetiology and Treatment. Front Oral Biol.Basel, Karger, 2012,vol 16,pp 19-31.

[2] Cassolato SF, Ross B, Daskalogiannakis J, Noble J, Tompson B, Paedo D. Treatment of dental anomalies in children with complete unilateral cleft lip and palate at SickKids hospital, Toronto. Cleft Palate Craniofac J., 2009 ;46(2):166-172.

[3] Sanjida Haque and Mohammad Khursheed Alam. Common Dental Anomalies in Cleft Lip and Palate Patients. Malays J Med Sci. ,2015, MarApr; 22(2): 55-60.

[4] Veerendra Prasad, Arun Kumar Singh, Vijay Kumar, Brijesh Mishra, Divya Narain Upadhyaya, Lakshmi Chandran Nair. Multidisciplinary treatment focussing on comprehensive orthodontic approach for improving facial esthetics in cleft lip and palate patients. Journal of Cleft Lip Palate and Craniofacial Anomalies, 2016, Vol : 3(1): 50-54.

[5] Mukut Seal, Pratim Talukdar, Kartik Pendharkar, Amritaksha Bhattacharyya. Treatment of an Open Apex with One-visit Apexification using Mineral Trioxide Aggregate. Int J Oral Care Res, 2016;4(2):142-145.

[6] Ivy Kiemle Trindade-Suedam, Bruno Felipe Gaia , Cheong Kuo Cheng, Paulo Alceu Kiemle Trindade, José Carlos Da Cunha Bastos , Beatriz Silva Câmara Mattos. Cleft Lip and Palate: Recommendations For Dental Anesthetic Procedure Based On Anatomic Evidences. J Appl Oral Sci. ,2012;20(1):122-7.

[7] Thorton, JB, dkk: The Incidence, Clasification, etiology, and embryology of oral cleft, Semin orthod. ,1996; 2(3): 162-168.

[8] Mohsen Shirazi, Homa Farhadifard, Meisam Moradi, and Hamid Golshahi. Multidisciplinary Treatment of Cleft Lip and Palate: A Case Report. Iran J Ortho. , 2015;10(2):e5054.

[9] Wilawan Weraarchakul, Wiboon Weraarchakul. Dental Caries in Children with Cleft Lip and Palate. J Med Assoc Thai. ,2017; 100 (Suppl. 6): S131-S135.

[10] Fabricio Guerrero, Asunción Mendoza, David Ribas, and Karla Aspiazu. Apexification: A systematic review. J Conserv Dent., 2018; 21(5): $462-465$.

[11] Shabahang S. Treatment options: apexogenesis and apexification. $J$ Endod. 2013;39(3 Suppl):S26-9.

[12] Parirokh M, Torabinejad M. Mineral trioxide aggregate: a comprehensive literature review-Part III: Clinical applications, drawbacks, and mechanism of action. $J$ Endod. 2010 Mar;36(3):400-13. 